\title{
Estimation of Sedimentary Basin Depth Using the Hybrid Technique for Gravity Data
}

\author{
Pham Thanh Luan, Do Duc Thanh ${ }^{*}$ \\ VNU University of Science, 334 Nguyen Trai, Thanh Xuan, Hanoi, Vietnam
}

Received 17 April 2017

Revised 22 May 2017; Accepted 05 June 2017

\begin{abstract}
In this paper we present the rapid method for determining the depth distribution of a sedimentary basin by combining the FFT-based and space domain techniques in gravity data interpretation. The method is tested on two 3D synthetic models which density contrast is constant and exponential variation with depth. Then, the method is applied to determine the depth distribution of Nam Con Son sedimentary basin in Vietnam. The obtained results coincide well with theoretical models and seismic data. The computation speed of the method is much faster than that of space domain technique.
\end{abstract}

Keywords: The hybrid technique, FFT-based technique, space domain technique, Nam Con Son basin.

\section{Introduction}

According to Parker [1], the computation time of gravity effect caused by a two-dimensional uneven layer of material using a FFT-based technique is proportional to $\mathrm{N} \ln (\mathrm{N})$, where $\mathrm{N}$ is the number of input and output points of model. For the same model, the computation time using a space domain technique is proportional to $\mathrm{N}^{2}$. The difference of computation time between two techniques is not so significant if the size of model is small. However, the number of calculations in the spacedomain technique increases rapidly compared to that in the FFT-based technique as the number of input and output points increases gradually.

Based on the FFT-based technique, Oldenburg [2] deduced an inversion method to compute a density contrast layer from its gravity anomalies. However, this method requires a given mean depth of the interface [2,3] and a low-pass filter to achieve convergence [2-6]. On the other hand, Bott [7] introduced another method using the space-domain technique despite the disadvantages of the computational speed. Bott's approach is based on the Bouguer slab formula that is readily adapted for the inversion procedure. In this paper, we combine both FFT-based and space-domain techniques in a model to solve their limitations and then apply to determine the depth distribution of Nam Con Son sedimentary basin in Vietnam.

\footnotetext{
*Corresponding author. Tel.: 84-902037545.

Email: doducthanh1956@gmail.com

https://doi.org/10.25073/2588-1124/vnumap.4203
} 


\section{Theory}

According to Parker [1], the vertical gravity effect due to an uneven, uniform layer of materialis:

$$
g_{z}=F^{-1}\left[-2 \pi \gamma \rho e^{\left(-|k| z_{0}\right)} \sum_{n=1}^{\infty} \frac{\llbracket \mathrm{k}]^{n-1}}{n !} F\left[h^{n}(r)\right]\right]
$$

where $\mathrm{F}\left[\right.$ ] is Fourier transform, $\mathrm{F}^{-1}[$ ] is inverse Fourier transform, $\gamma$ is the gravitational constant, $\rho$ is the density contrast, $\mathrm{k}$ is the wave number and $\mathrm{z}_{0}$ is the mean depth of the horizontal interface. Depth to the interface is defined by the equation $\mathrm{z}=\mathrm{h}(\mathrm{r})$.

Based on the Parker algorithm, Oldenburg [2] deduced a method to compute the depth to the undulating interface from the gravity anomaly profile. Equation (1) can be rearranged as:

$$
h(r)=F^{-1}\left[-\frac{F\left[g_{z}\right] e^{|k| z_{0}}}{2 \pi y \rho}-\sum_{n=2}^{\infty} \frac{\mid k]^{n-1}}{n !} F\left[h^{n}(r)\right]\right]
$$

However, the convergence of Oldenburg's inversion procedure can be guaranteed only after a lowpass filter has to be used. This filter is defined by:

$$
B(k)=\frac{1}{2}\left[1+\cos \left(\frac{1}{2(S H-W H)}\right)\right], W H \leq \begin{array}{r}
|k / 2 \pi|<W H \\
|k / 2 \pi| \leq S H \\
|k / 2 \pi|>S H
\end{array}
$$

where $\mathrm{WH}$ and $\mathrm{SH}$ are frequency parameters. The filter cuts off the frequencies higher than $\mathrm{SH}$ and the frequencies lower than WH will be fully passed, while the frequencies between WH and SH will be partly passed.

Detection of WH and $\mathrm{SH}$ values is quite difficult and the depth of interface is smoothed when using Parker-Oldenburg method. Therefore, we used the hybrid technique that based on Bott's[7] approach to calculate the depth. Following Bott, the first approximation of the depth to basement is:

$$
h_{0}=\frac{g_{z}}{2 \pi \gamma \rho}
$$

The gravity anomalies of this surface are then calculated by the FFT-based technique. The calculated anomaly is compared with the observed anomaly. Using the difference between calculated and observed anomaly, the depth distribution of interface can be improved as follows:

$$
\begin{aligned}
& \Delta h_{i}=\frac{\Delta g_{z}}{2 \pi \gamma \rho} \\
& h_{i+1}=h_{i}+\Delta h_{i}
\end{aligned}
$$

This procedure can be repeated until the model is satisfied due to convergence is met.

In the case of sedimentary basins which the density contrast varies exponentially with depth $\rho=\rho_{0} e^{-\lambda z}$, the gravity effect of basin can be calculated by Granser [8] method:

$$
\begin{aligned}
& g_{z}=\frac{2 \pi \gamma \rho_{0}}{\lambda}\left(1-e^{-\lambda z_{0}}\right)+2 \pi \gamma \rho_{0} e^{-\lambda z_{0}} \\
& \times F^{-1}\left[\frac{e^{-|k| z_{0}}}{|k|+\lambda}\left(F\left[1-e^{(-\lambda \Delta \mathrm{h})}\right]-\sum_{n=1}^{\infty} \frac{(-|\mathrm{k}|)^{n}}{n !} F\left[e^{-\lambda \Delta h} \Delta h^{n}\right]\right)\right]
\end{aligned}
$$

where $\Delta \mathrm{h}=\mathrm{h}(\mathrm{r})-\mathrm{z}_{0}$

Equations (3) and (4) can be expressed as 


$$
\begin{aligned}
& h_{0}=-\frac{1}{\lambda} \ln \left(1-\frac{\lambda g_{z}}{2 \pi \gamma \rho_{0}}\right) \\
& \Delta h_{i}=-\frac{1}{\lambda} \ln \left(1-\frac{\lambda \Delta g_{z}}{2 \pi y \rho_{0} \mathrm{e}^{-\lambda h_{\mathrm{i}}}}\right)
\end{aligned}
$$

\section{Numerical examples}

We use two sedimentary basin models with the same depth for testing. The parameters of models are as follows: the total number of columns and rows are $112 \times 112$; the square grid interval is $1 \mathrm{~km}$. The depth distribution of the interface is shown in Figure la.

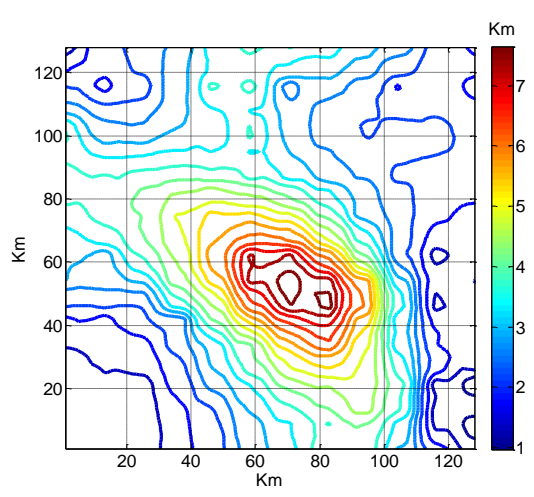

(a)

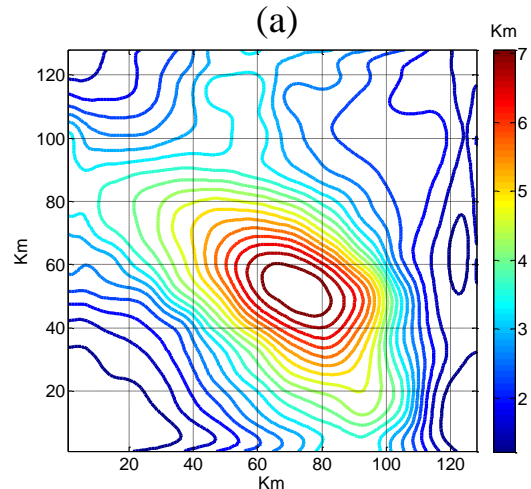

(d)

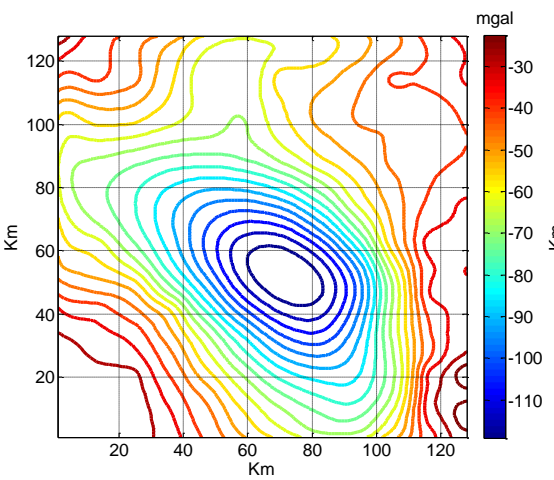

(b)

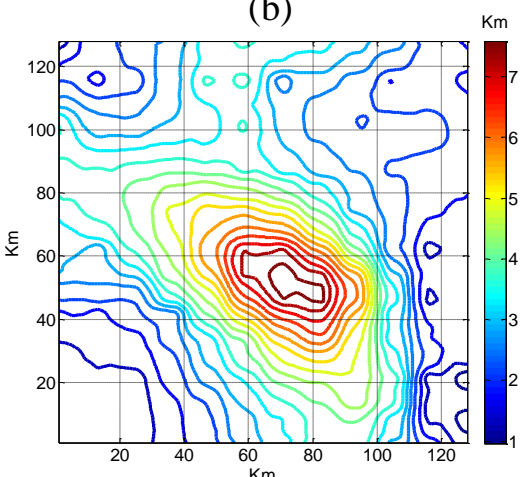

(e)

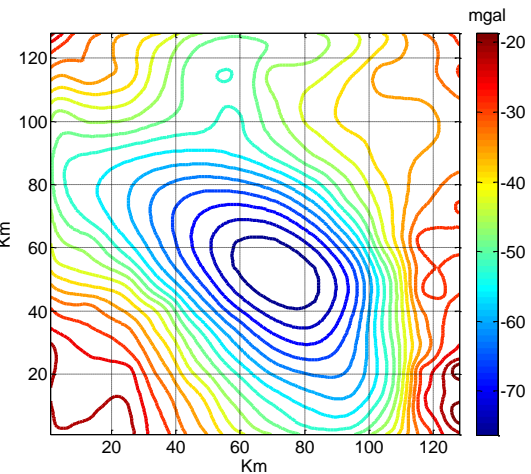

(c)

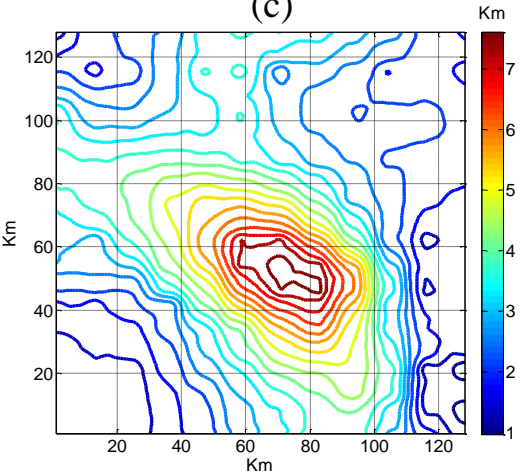

(f)

Figure 1. Numerical examples.

(a) The model depth, (b) Gravity anomalies due to basin with density contrast $-0.48 \mathrm{~g} \mathrm{~cm}^{-3}$, (c) Gravity anomalies due to basin with exponential density contrast $-0.48 \mathrm{e}^{-0.15 z} \mathrm{~g} \mathrm{~cm}^{-3}$, (d) Inversed depth of model with density contrast $-0.48 \mathrm{~g}$ $\mathrm{cm}^{-3}$ using Parker-Oldenburg method, (e) Inversed depth of model with density contrast $-0.48 \mathrm{~g} \mathrm{~cm}^{-3}$ using the hybrid technique, (f) Inversed depth of model with exponential density contrast $-0.48 \mathrm{e}^{-0.15 z} \mathrm{~g} \mathrm{~cm}^{-3}$ using the hybrid technique

The first example has density contrast is constant $\rho=-0.48 \mathrm{~g} \mathrm{~cm}^{-3}$. With this example, the gravity anomaly obtained from the application of the FFT-based technique is shown in Figure $1 b$. Using this field, we performed the inverse procedure by using both methods Parker-Oldenburg and the hybrid 
technique. The results are shown in Figure 1d, e. According to Oldenburg, a low-pass filter has to be used to achieve convergence and this is the reason why the resulting interface is smoothed (Figure 1d). As a result, the root mean square $(R m s)$ of depth is quite large, $R m s$ is equal to $0.2166 \mathrm{~km}$. Otherwise, when using the hybrid technique, the inversed depth is improved much (Figure 1e). Here, the inversed depth result compares very favorably with the depth model and $R m s$ equals to $0.0152 \mathrm{~km}$.

The second example has density contrast varies exponentially with depth $-0.48 \mathrm{e}^{-0.15 \mathrm{z}} \mathrm{g} \mathrm{cm}^{-3}$. The gravity anomaly of this model has been calculated by Granser's formula (Figure 1c). Then we only used the hybrid technique to calculate the depths to the interface. The obtained result is shown in Figure If. The result shows that, the inversed depth coincides well with the model depth. In this case, Rms equals to $0.0158 \mathrm{~km}$.

Using the hybrid technique, the computation time for each model is about 9 seconds. For equivalent space-domain calculations on these models, the computation time is about 6 hours.

\section{Field example}

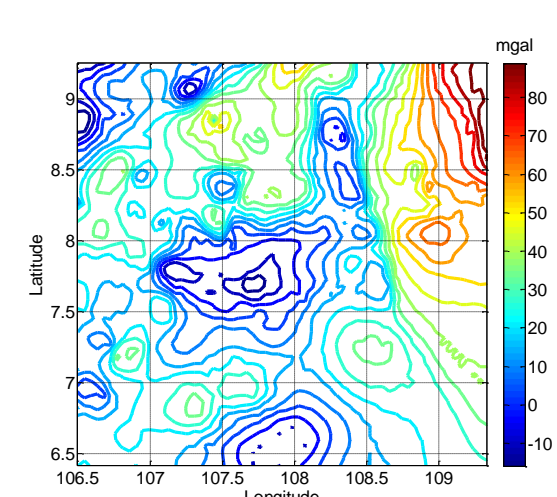

(a)

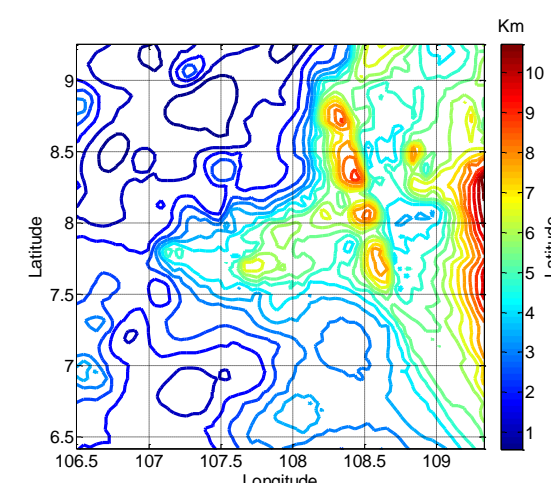

(d)

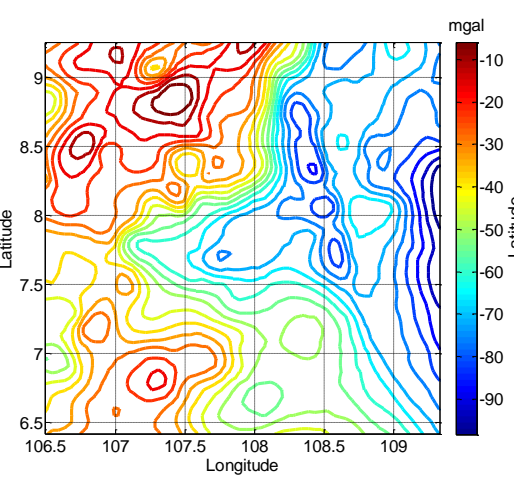

(b)

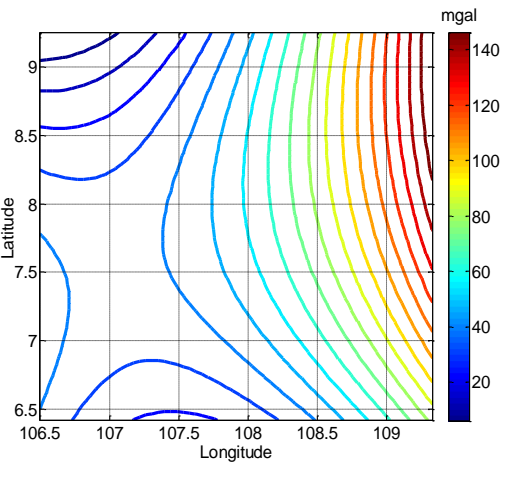

(c)

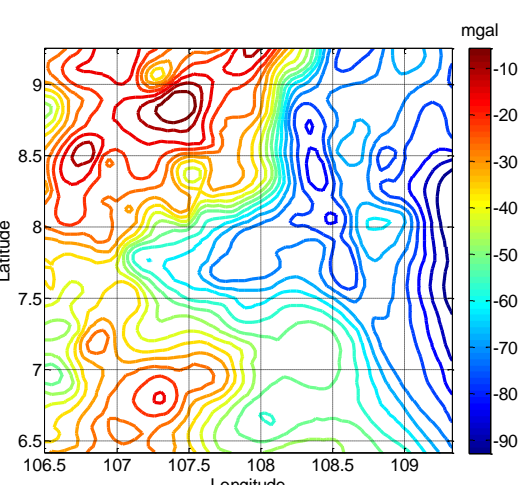

(e)

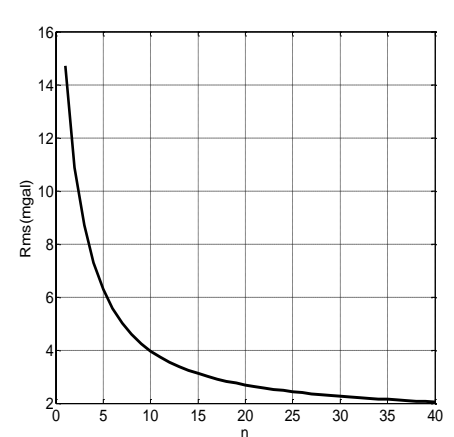

(f)

Figure 2. Nam Con Son sedimentary basin.

(a) Bouguer gravity anomaly, (b) Residual gravity, (c)Regional effect,(d) Inversed depth of basin,

(e) The gravity anomaly at the last iteration, (f) Rate of convergence 
The hybrid technique is also applied in gravity field to determine the depth distribution of Nam Con Son sedimentary basin. Study area is about $100,000 \mathrm{~km}^{2}$, ranging from $9^{0} \mathrm{~N}$ to $11^{0} \mathrm{~N}$ latitude and $106.5^{\circ} \mathrm{E}$ to $109.5^{\circ} \mathrm{E}$ longitude. The results are shown in Figure 2.

Figure $2 a$ is the Bouguer gravity anomaly map presented by Bui Cong Que and Nguyen Hiep [9]. Figure $2 b$ illustrates the residual gravity of Nam Con Son sedimentary basin obtained from the Bouguer gravity anomalies by removing the regional effect (Figure 2c). Residual density of basin is $\rho=-0.5 e^{-0.161 z}$ which was determined from density data of drillhole 21S - 1X [9].

Figure $2 d$ shows the depth result of Nam Con Son sedimentary basin. Figure $2 e$ shows gravity anomaly at the last iteration. By compared Figure $2 e$ and Figure $2 b$, it is clearly that the depth model of Nam Con Son sedimentary basin obtained from the hybrid technique produces the gravity anomaly very similar in shape to the residual gravity. The rate of convergence of method is fast, and it only takes 5 seconds for calculation. The depths to the interface compare favorably with the results of seismic exploration methods [9].

\section{Conclusions}

The computation results obtained from numerical and field examples showed that the hybrid technique based on the FFT and space domain techniques would be a useful approach in gravity interpretation, especially in inversion procedure. According to the technique, the gravity anomaly is easily computed from Parker and Granser's formula. Then, the depth to the interface is determined by Bott's approach. The obtained results compare very favorably with theoretical models and seismic data. The computation speed of the hybrid technique is much faster than that of space domain technique. Moreover, the depth results using hybrid technique are also more accurate than those using Parker-Oldenburg method.

\section{References}

[1] R. L. Parker, The Rapid Calculation of Potential Anomalies, Geophys. J. R. astr. SOC. 31 (1972) 447-455.

[2] Oldenburg, The inversion and interpretation of gravity anomalies, Geophysics, 39 (1974) 526-536.

[3] David Gómez Ortiz, Bhrigu N.P. Agarwal, 3DINVER.M: A MATLAB program to invert the gravity anomaly over a 3D horizontal density interface by Parker-Oldenburg's algorithm, Computers \& Geosciences, 31 (2005) $513-520$

[4] Young Hong Shin, Kwang Sun Choi, HouzeXu, Three-dimensional forward and inverse models for gravity fields based on the Fast Fourier Transform, Computers \& Geosciences, 32 (2006) 727-738.

[5] R. Nagendra, P.V.S. Prasad, V.L.S. Bhimasankaram, Forward and inverse computer modeling of gravity field resulting from a density interface using Parker-Oldenberg method, Computers \& Geosciences, 22 (1996) 227 231.

[6] R. Nagendra, P.V.S. Prasad, V.L.S. Bhimasankaram, FORTRAN program based on Granser's algorithm for inverting a gravity field resulting from a density interface, Computers \& Geosciences, 2 (1996) 219-225.

[7] Bott, M.H.P, The use of rapid digital computing methods for direct gravity interpretation of sedimentary basins, Geophysical Journal of the Royal Astronomical Society, 3(1960)63-67.

[8] Harald Granser, Three - dimensional interpretation of gravity data from sedimentary basins using an exponential density - depth function, Geophysical Prospecting, 35(1987)1030 - 1041.

[9] Bui Cong Que and Nguyen Hiep, Geophysical field characteristics of Vietnam continental shelf and its neighbor, Project final report No. 48-B.03.02, 48-B marine research program, Hanoi, 1990. (in Vietnamese) 